\title{
MEASURABILITY OF PARTIAL DERIVATIVES
}

\author{
MOSHE MARCUS AND VICTOR J. MIZEL ${ }^{1}$ \\ ABSTRACT. Let $f$ be a real function defined in $R_{n}$. In this note we give a \\ sufficient condition in order that the set of points where the partial derivative \\ $\partial f / \partial x_{i}$ exists is Lebesgue measurable and $\partial f / \partial x_{i}$ is a measurable function on \\ this set. This result unifies and extends a number of previous results.
}

1. According to a classical result due to Banach, a real Lebesgue measurable function $f$ on the real line has the following property. The set of points $\Omega(f)$ where the derivative $f^{\prime}$ exists is measurable and $f^{\prime}$ is a measurable function on $\Omega(f)$. This result has an extension to functions defined in $R_{n}$. If $f$ is a real, Lebesgue measurable function on $R_{n}$ then the set of points $\Omega(f)$ where $f$ is differentiable is measurable and the partial derivatives of $f$ are measurable on $\Omega(f)$. This result is due to Haslam-Jones [1]. A simple proof (due to Federer) can be found in [3, p. 268]. However, the set of points where a given first-order partial derivative of $f$ exists need not be measurable. For an example see [2, §7]. In this note we are concerned with conditions on $f$ which ensure that the partial derivatives are measurable.

Let $x=\left(x_{1}, \ldots, x_{n}\right)$ denote a generic point in $R_{n}$. Let $J$ be an open $n$ dimensional interval and let $f$ be a real measurable function in $J$. (In this note the term "measurable" and all related terms will refer to Lebesgue measure.) We denote by $\Omega_{i}(f)$ the set of points $\left\{x \in J:\left(\partial f / \partial x_{i}\right)(x)\right.$ exists and is finite $\}$.

The following results are known.

I. If $f$ is continuous on $\tau \cap J$ for almost every line $\tau$ parallel to the $x_{i}$ axis then $\Omega_{i}(f)$ is measurable and $\partial f / \partial x_{i}$ ismeasurable on $\Omega_{i}(f)$.

II (Serrin [2]). If $f$ is monotone on $\tau \cap J$ for almost every line $\tau$ parallel to the $x_{i}$ axis then $\partial f / \partial x_{i}$ exists and is finite a.e. in $J$.

III (Serrin [2]). If $f$ is of bounded variation on $\tau \cap J$ for almost every line $\tau$ parallel to the $x_{i}$ axis then there exists an equivalent function $h$ such that $\partial h / \partial x_{i}$ exists and is finite a.e. in $J$.

The last result was obtained as a corollary of II by showing that a measurable function $f$ satisfying the condition of III is equal a.e. to the difference of two measurable functions satisfying the condition of II.

The purpose of this note is to present another sufficient condition for the measurability of partial derivatives which provides a considerable extension of the results I--III.

Received by the editors October 1, 1976.

AMS (MOS) subject classifications (1970). Primary 26A54.

I Partially supported by the U.S. National Science Foundation under Grant MPS 71-02776. 
2. We start with

DEFINITION 1. Let $g$ be a real function defined on an open interval I of the real line. If $t_{0} \in I$ we set

$$
\begin{array}{ll}
L^{+} g\left(t_{0}\right)=\limsup _{t \rightarrow t_{0}+0} g(t), & L^{-} g\left(t_{0}\right)=\limsup _{t \rightarrow t_{0}-0} g(t), \\
l^{+} g\left(t_{0}\right)=\liminf _{t \rightarrow t_{0}+0} g(t), & l^{-} g\left(t_{0}\right)=\liminf _{t \rightarrow t_{0}-0} g(t) .
\end{array}
$$

We shall say that $g$ satisfies the (SM) condition at $t_{0}$ if,

$$
\min \left(L^{+} g\left(t_{0}\right), L^{-} g\left(t_{0}\right)\right) \leqslant g\left(t_{0}\right) \leqslant \max \left(l^{+} g\left(t_{0}\right), l^{-} g\left(t_{0}\right)\right) .
$$

Note that if $g$ is monotone in a neighborhood of $t_{0}$ or if $g$ is right (or left) continuous at $t_{0}$, then $g$ satisfies the (SM) condition at $t_{0}$. However, the (SM) condition does not imply either of the preceding two. The following is our main result.

THEOREM 1. Let $f$ be a real measurable function on an open $n$-dimensional interval J. Suppose that for almost every line $\tau$ parallel to the $x_{i}$ axis the restriction $\left.f\right|_{\tau \cap J}$ satisfies the (SM) condition everywhere in $\tau \cap J$. Then $\Omega_{i}(f)$ is measurable and $\partial f / \partial x_{i}$ is a measurable function on $\Omega_{i}(f)$.

Proof. Let $\delta_{h}^{i} f=h^{-1}\left[f\left(x+h e^{i}\right)-f(x)\right]$, where $h$ is real and different from zero and $e^{i}$ is the unit vector in the direction of the $x_{i}$ axis.

Set

$$
D_{i}^{\mathrm{rat}} f(x)=\lim _{\substack{r \rightarrow 0 \\ r \text { rational }}} \delta_{r}^{i} f(x),
$$

for every $x$ in $J$ where the limit exists. Let $\Omega_{i}^{\prime}(f)$ denote the set of points in $J$ where $D_{i}^{\text {rat }} f$ exists and is finite. Clearly, $\Omega_{i}^{\prime}(f)$ is measurable and $D_{i}^{\text {rat }} f$ is a measurable function on this set. Furthermore,

$$
\Omega_{i}(f) \subseteq \Omega_{i}^{\prime}(f) \text { and }\left(\partial f / \partial x_{i}\right)(x)=D_{i}^{\mathrm{rat}} f(x), \quad x \in \Omega_{i}(f) .
$$

Let $\tau$ be a line parallel to the $x_{i}$ axis such that $\left.f\right|_{\tau \cap J}$ satisfies the (SM) condition everywhere in $\tau \cap J$. We shall show that

$$
\Omega_{i}(f) \cap \tau \supseteq \Omega_{i}^{\prime}(f) \cap \tau .
$$

Let $x$ be a point of $\Omega_{i}^{\prime}(f) \cap \tau$ and let $\left\{h_{m}\right\}_{1}^{\infty}$ be a sequence converging to zero such that $h_{m} \neq 0$ for all $m$. Applying the (SM) condition at each of the points $x+h_{m} e^{i}(m=1,2, \ldots)$ one can choose two sequences of rational numbers $\left\{r_{m}\right\}$ and $\left\{r_{m}^{\prime}\right\}$ such that

$$
r_{m} / h_{m} \rightarrow 1 \text { and } r_{m}^{\prime} / h_{m} \rightarrow 1 \text { as } m \rightarrow \infty
$$

and 


$$
\begin{aligned}
f\left(x+r_{m}^{\prime} e^{i}\right) & >f\left(x+h_{m} e^{i}\right)-h_{m} / m, \\
f\left(x+r_{m} e^{i}\right) & <f\left(x+h_{m} e^{i}\right)+h_{m} / m, \quad m=1,2, \ldots
\end{aligned}
$$

From (7) we obtain

$$
\frac{r_{m}}{h_{m}} \delta_{r_{m}}^{i} f(x)-\frac{1}{m}<\delta_{h_{m}}^{i} f(x)<\frac{r_{m}^{\prime}}{h_{m}} \delta_{r_{m}^{\prime}}^{i} f(x)+\frac{1}{m}, \quad m=1,2, \ldots
$$

Letting $m$ tend to infinity we get

$$
\lim _{m \rightarrow \infty} \delta_{h_{m}}^{i} f(x)=D_{i}^{\mathrm{rat}} f(x) .
$$

Since $\left\{h_{m}\right\}$ was an arbitrary sequence in $R \backslash\{0\}$, converging to zero, we deduce that $\left(\partial f / \partial x_{i}\right)(x)$ exists. This proves (5). In view of our assumptions, (4) and (5) imply the conclusion of the theorem.

REMARK. It is clear from the proof that the conclusion of the theorem remains valid if we merely require that the (SM) condition holds at all but a finite number of points on $\tau \cap J$ for almost all lines $\tau$ parallel to the $x_{i}$ axis. However, if "finite" is replaced by "countable" the conclusion of the theorem may fail. This is shown by a counterexample given in $[2, \S 7]$.

Clearly, results I and II follow immediately from Theorem 1. To prove III one may argue as follows. Let $f$ be a function as in III and set

$$
h(x)=\lim _{m \rightarrow \infty} f\left(x+m^{-1} e^{i}\right) .
$$

Then $h$ is measurable in $J$. If $\tau$ is a line parallel to the $x_{i}$ axis such that $\left.f\right|_{\tau \cap J}$ is of bounded variation, then $\left.h\right|_{\tau \cap J}=\left.f\right|_{\tau \cap J}$ a.e. (with respect to linear Lebesgue measure) and $\left.h\right|_{\tau \cap J}$ is everywhere right continuous. Hence, $h=f$ a.e. in $J$ and $\left.h\right|_{\tau \cap J}$ satisfies the (SM) condition everywhere in $\tau \cap J$ for almost all lines $\tau$ parallel to the $x_{i}$ axis. Thus Theorem 1 implies III.

\section{REFERENCES}

1. U. S. Haslam-Jones, Derivative planes and tangent planes of a measurable function, Quart. J. Math. Oxford 3 (1932), 120-132.

2. J. Serrin, On the differentiability of functions of several variables, Arch. Rational Mech. Anal. 7 (1961), 359-372. MR 25 \#3131.

3. E. M. Stein, Singular integrals and differentiability properties of functions, Princeton Univ. Press, Princeton, N. J., 1970. MR 44 \# 7280.

Department of Mathematics, Technion, Israel Institute of Technology, Haifa, Israel

Department of Mathematics, Carnegie-Mellon University, Pittsburgh, Pennsylvania 15213. 